\title{
Forest Monitoring and Wildland Early Fire Detection by a Hierarchical Wireless Sensor Network
}

\author{
Antonio Molina-Pico, ${ }^{1}$ David Cuesta-Frau, ${ }^{1}$ Alvaro Araujo, ${ }^{2}$ \\ Javier Alejandre, ${ }^{3}$ and Alba Rozas ${ }^{2}$ \\ ${ }^{1}$ Technological Institute of Informatics, Polytechnic University of Valencia, Alcoy Campus, Plaza Ferrándiz y Carbonell 2 , \\ 03801 Alcoy, Spain \\ ${ }^{2}$ Polytechnic University of Madrid, Campus Ciudad Universitaria, Avenida Complutense 30, 28040 Madrid, Spain \\ ${ }^{3}$ ISDEFE S.A., Beatriz de Bobadilla 3, 28040 Madrid, Spain
}

Correspondence should be addressed to David Cuesta-Frau; dcuesta@disca.upv.es

Received 21 October 2015; Revised 11 January 2016; Accepted 18 January 2016

Academic Editor: Kewei Zhang

Copyright (C) 2016 Antonio Molina-Pico et al. This is an open access article distributed under the Creative Commons Attribution License, which permits unrestricted use, distribution, and reproduction in any medium, provided the original work is properly cited.

\begin{abstract}
A wildland fire is an uncontrolled fire that occurs mainly in forest areas, although it can also invade urban or agricultural areas. Among the main causes of wildfires, human factors, either intentional or accidental, are the most usual ones. The number and impact of forest fires are expected to grow as a consequence of the global warming. In order to fight against these disasters, it is necessary to adopt a comprehensive, multifaceted approach that enables a continuous situational awareness and instant responsiveness. This paper describes a hierarchical wireless sensor network aimed at early fire detection in risky areas, integrated with the fire fighting command centres, geographical information systems, and fire simulators. This configuration has been successfully tested in two fire simulations involving all the key players in fire fighting operations: fire brigades, communication systems, and aerial, coordination, and land means.
\end{abstract}

\section{Introduction}

Forest fires are a recurrent phenomenon, natural or manmade, in many parts of the world. Vulnerable areas are mainly located in temperate climates where pluviometry is high enough to enable a significant level of vegetation, but summers are very hot and dry, creating a dangerous fuel load. Global warming will contribute to increase the number and importance of these disasters. Every season, not only are thousands of forest hectares destroyed by wildland fires, but also assets, properties, and public resources and facilities are destroyed. Moreover, firefighter and civilians are at risk, with a terrible toll in human lives each year.

Although progress has been made in the field of wildfire fighting in the last decades, there is still a need to strengthen the disaster response capacity, including early warning systems and improvements in real time exchange of data at all stages and levels of a forest monitoring scheme. Technological breakthroughs will be a key force driving change in wildland fire fighting. Recent developments in information and communication technologies are already having a huge impact, specially forest fire detection systems [1].

There are systems based on satellite imagery [2], but they are not used in real time applications due to their long scanning cycle [3], poor resolution, and cost. Other systems based on short range images have been proposed, using either opti$\mathrm{cal}$, infrared, or thermal images. However, these approaches are very outlier sensitive: direct and strong sunlight, insufficient light, or smoke. They are also very false alarm prone. Radio acoustic sounding has also been proposed as a way to infer the meteorological flow or temperature profiles in forest areas [4]. Nevertheless, it lacks resolution, it is expensive (radars and acoustics sources required), and it is susceptible to interferences, such as wind direction changes.

Other recent works describe detection systems based on distributed wireless sensor networks (WSNs). In [5], a WSN 


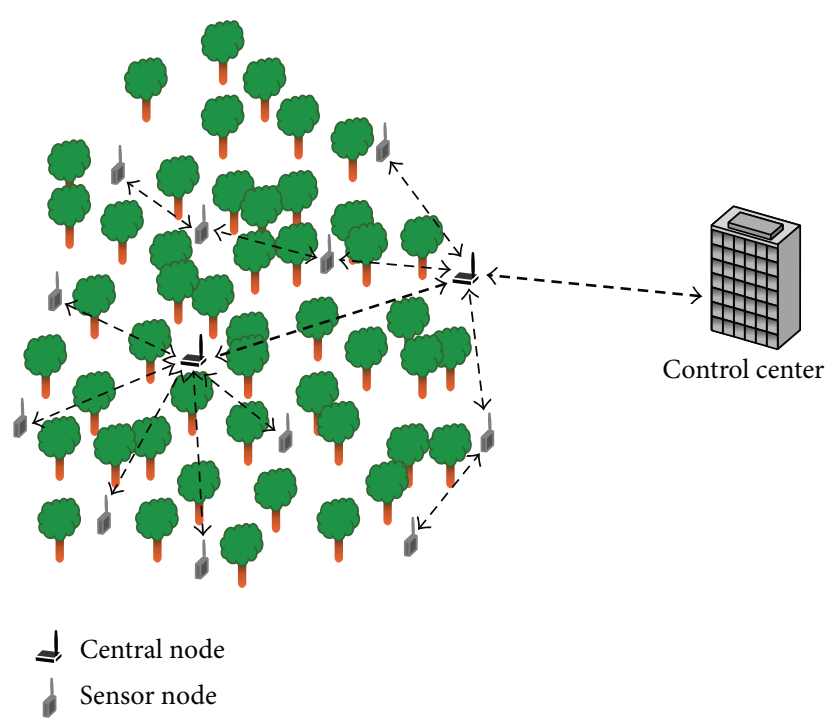

FIGURE 1: General structure of the approach proposed. Sensor nodes capture data from the environment that are uploaded to the central nodes, which transfer all the information to the system middleware.

based on cameras, fire detectors, and $802.11 \mathrm{~g}$ access points is described. This approach is aimed at detecting and verifying fires in rural and forest environments, and it sends alarms to a central server. The work in [3] proposes a WSN based on ZigBee, GPRS, and Ethernet communication modules. Ganesh et al. [6] describe a forest fire detection system based on WSN and on solar energy harvesting modules. It applies a node hopping scheme to reach the data server.

Our approach is also based on the WSN paradigm but has been designed and developed in the context of a research project that included all the key actors in forest fire fighting operations: engine, hand, and helitack crews, satellite operators, forest services agencies, information and communication technologies providers, management organizations (wildland fire management, fire operations, fuel management, and aviation management), authorities, aviation services, airtankers, weather services agencies, and burned area rehabilitators. This unique research ecosystem has provided our solution with a holistic perspective that results in a set of distinguishing features:

(i) no need for a preinstalled communications network: most schemes need to have GSM coverage or WiFi to work. This approach assumes that there is no communication network available at the monitoring zone and creates its own network;

(ii) time synchronization: all measurements taken must have a time-stamp, but some wireless nodes have no way to get the current time by themselves. The network is able to get the current time from the GPS module and correct time deviations;

(iii) dual functionality, that is, environment monitoring and fire early detection: deployment of networks can be costly for the only purpose of fire detection. Even at high risk areas, the probability of fire may be very low, and a WSN can be underexploited and become a waste of resources. Environment monitoring contributes to optimize the usage of the network;

(iv) not being based on cameras/images: video or image acquisition and transmission are very demanding in terms of bandwidth and power consumption. This approach is very suitable for ground based monitoring stations, but not for general "deploy and forget" approaches;

(v) integration with the operation centre: the network proposed includes a middleware layer to share data with command centres and other wildland fire fighting units. This enables a full picture of the emergency in real time.

The general structure of our approach is depicted in Figure 1. The node structure is composed of 2 levels, central nodes, devoted mainly to short and long range communications and control purposes, and sensor nodes, used to collect data from the area under monitoring and send them to the central nodes. All node types can include environment and meteorological sensors.

\section{Materials and Methods}

The main design and functional requirements of the WSN were

(i) contribution to the forest fire fighting strategy, providing critical data to improve their safety and efficiency;

(ii) providing of valuable forest and climate parameters input to the fire propagation models. This included temperature, humidity, rainfall, wind, smoke, and solar radiation; 


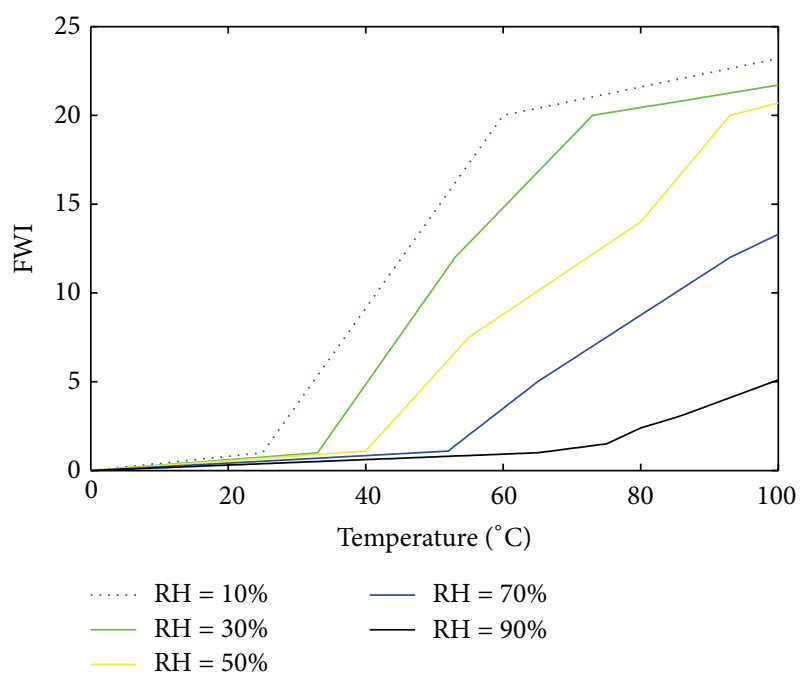

FIGURE 2: Sensitivity of the FWI to temperature.

(iii) early detection of forest fires, alarm management, and real time reporting about the fire evolution. They provide alarms by integrating information from several nodes;

(iv) following of a "deploy and forget" policy. Nodes will not need any kind of maintenance or battery recharging. Energy harvesting techniques and an optimized low power consumption will ensure uninterrupted functioning.

Fire risk is computed according to the Fire Weather Index (FWI) System. This system was developed by the Canadian Forest Service (CFS), which is based on several decades of forestry research. The FWI System estimates the moisture content of three different fuel classes using weather observations. The fuel class which is of our interest is the Fine Fuel Moisture Code (FFMC). This code is used to indicate ease of ignition and it is computed with the temperature, relative humidity, wind, and rain. Figure 2 represents the sensitivity of the FWI to temperature.

Other requirements corresponded to standard WSN technical specifications: sensors and nodes had to be inexpensive, small, and energy efficient. They had to be deployed and configured easily and respect the natural environment. The network algorithms and protocols were based on procedures that enabled self-organization, reconfiguration, and dynamic adaptation.

In order to successfully address these requirements, we adopted a standard wireless sensor network approach using two levels for the nodes, central nodes and sensor nodes, and some optimization techniques to minimize power requirements. During network deployment, each sensor would record its geolocalisation based on GPS information. In addition, RSSI data was also collected and processed for selfawareness network arrangement. This RSSI information can be complementary to the absolute GPS position or can be the only data available in terms of geographical information.

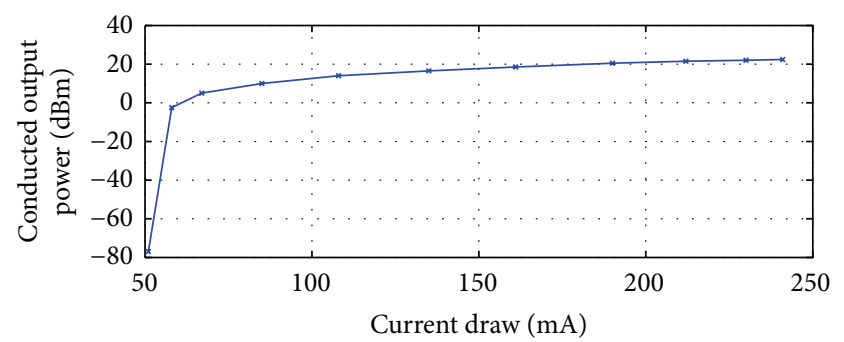

FIGURE 3: AC4868-250M conducted output power versus current drawn. The current is set by suitable EEPROM configuration $(x$ points).

The nodes can also be deployed in vehicles. This provides additional functionality; since power requirements will be less demanding for such nodes, long range communications will be easier, and the network is equipped with some mobility degree.

2.1. Central Nodes (CNs). The CNs have more powerful communication and computation capabilities than the sensor nodes. Their main role is to gather and cluster data from the node sensors, manage alarms and commands, and build the core network. They can optionally also include sensing capabilities.

The processing unit is based on a PIC24FJ256GB110. This is a powerful microcontroller that fulfills the technical requirements of the WSN in terms of memory, input/output ports, and communication interfaces. The internal voltage was set as $2.5 \mathrm{~V}$. The CPU includes an internal regulator that converts the battery voltage into $3.3 \mathrm{~V}$. The CPU uses two external oscillators. One provides the required $8 \mathrm{MHz}$ clock for the system, and the second one generates the real time clock. Power is supplied by rechargeable batteries connected to small solar panels for energy harvesting.

Each $\mathrm{CN}$ will account for an area of a risk map previously defined for the interest zone. The network area covered by a CN can be updated dynamically. A subset of the $\mathrm{CN}$ will directly be linked to the Control Centre or command centre, defined as the main central nodes.

Their communication range will span several kilometers to guarantee a reliable service by means of ISM communication. The ISM transceiver module is the AC4868-250 from Laird Technologies. It operates under European ETSI regulations for the $868-870 \mathrm{MHz}$ band and the network topology may be a point-to-point, point-to-multipoint, or peer-to-peer architecture. The maximum allowed output power is $250 \mathrm{~mW}$ and it may reach up to $15 \mathrm{~km}$ in line of sight. Since in most real applications nodes will not be separated that much, the output power may be set as low as desired to save power consumption. Figure 3 shows the relationship between the conducted output power and the current drawn. The radiated output power is achieved with the addition of a $2 \mathrm{dBi}$ antenna.

They also include a GSM/GPRS module just in case there is a cellular data link available in the area, although some forest areas are not covered by this service. A ZigBee 


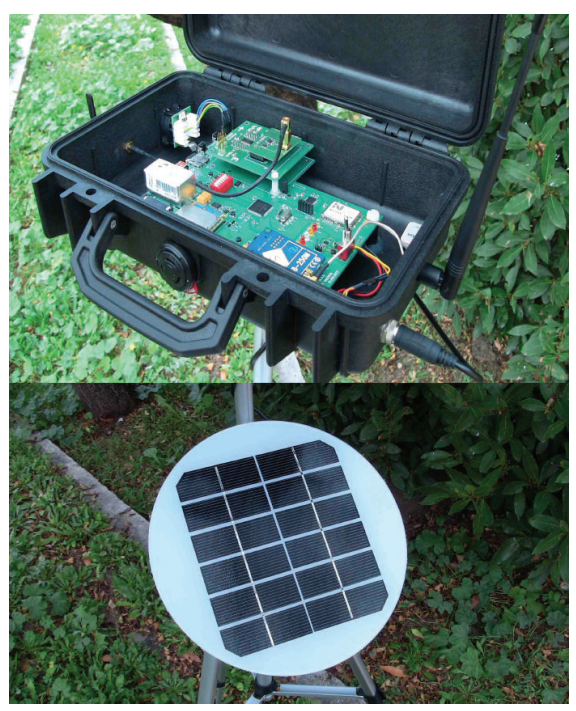

FIGURE 4: Image of an actual CN deployed in a forest area, including the solar panel used for energy harvesting.

module is used to communicate with the sensor nodes, and a geolocalisation module can be included to account for the exact position of the CNs. These nodes will also have the capability of playing the role of a Control Centre, by direct connection to a computer or a graphic visualisation system.

The CNs have three operational modes:

(i) Trunk Link Search Mode (TLSM): this mode is employed by new central nodes to be included in the network dynamically;

(ii) Test/Reset Mode (TRM): this is an internal test mode to check the node status. It can be entered periodically or by means of a command from the Control Centre;

(iii) Operative Mode (OPM): this is the normal operation mode. All the data from the sensor nodes are collected and processed; there is a permanent link with the Control Centre, and the commands are managed and dispatched.

These nodes are deployed in areas populated by a suitable number of sensor nodes in order to provide them with the communications and management services required. An example of a real implementation and deployment of a $\mathrm{CN}$ is depicted in Figure 4. In real applications, it is convenient to deploy the central nodes a short distance away from the monitoring area and to place them in an elevated location. This improves energy harvesting, long range communications and avoids vandalism.

2.2. Sensor Nodes (SNs). The SN network is in charge of monitoring the environmental and weather variables related to forest fires' onset risk and propagation and to provide early alarms based on these parameters. In particular, SN monitors the air temperature, its relative humidity, wind speed and direction, and $\mathrm{CO}$ and $\mathrm{CO}_{2}$ levels. The $\mathrm{SN}$ network is composed of several end nodes with the usual characteristics of wireless sensor networks devices: (i) processing unit: it consists of a small ultralow power microcontroller from the Texas Instruments MSP430 family. It is specifically designed for energy efficient applications, which is the main reason for its inclusion in this approach;

(ii) communications system: it is featured by a radio transceiver and its corresponding radio frequency stage. In particular, the transceiver model is the CC1101 part, also from Texas Instruments. The field network operates on the $433 \mathrm{MHz}$ radio region, an unlicensed ISM frequency band. The reason for working on this specific frequency region has to do with its high transmission range and the fact that it is relatively unused in forest environments;

(iii) sensing module: it is composed of several monitoring devices. Every end node has a relative humidity and temperature integrated sensor, SHT75 from Sensirion. It can record the air temperature with an accuracy of \pm 0.3 and its relative humidity with a maximum error of $\pm 1.8 \%$. It also includes gas/smoke sensors. In addition, some special nodes have an attached pole, with an anemometer and a vane for measuring wind speed and direction and a precipitation sensor. The design and structure of the SNs enable their customisation for any other sensing need.

The dimensions of the SNs are approximately $10 \times 5 \times$ $2 \mathrm{~cm}$. They are powered from a small lithium coin battery with a capacity of $600 \mathrm{mAh}$. The communication protocols and power saving modes were optimized to guarantee at least 2 years of uninterrupted functioning. An example of a SN deployment is shown in Figure 5.

The SNs can be in different functioning modes, depending on the occurrence of a predefined event. They can only be in a single mode, and not all the nodes can be in any mode, 


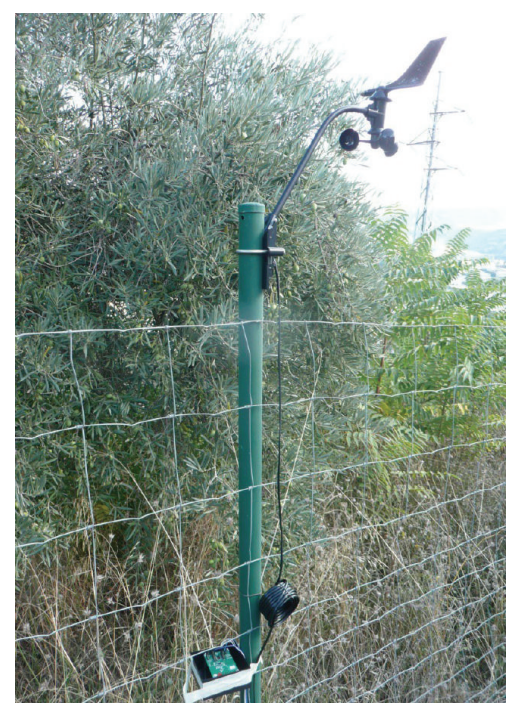

FIGURE 5: Image of a sensor node located at the boundaries of an area of interest, including an anemometer.

since it depends on the configuration and external conditions of each node. The main modes for the SNs are

(i) Link Search Mode (LSM): this mode enables the connection to other nodes becoming a node of the entire network;

(ii) Test/Reset Mode (TRM): this is a self-test mode to report about the node status to higher level nodes;

(iii) Automatic Monitoring Mode (AMM): this is the usual working mode when there is no alarm. Data are collected periodically and sent to the central nodes, using an optimized algorithm to minimize network traffic, memory requirements, and power consumption;

(iv) Fire Alarm Mode (FAM): this mode is reached if the node itself detects a fire and receives an alarm from a central node or an alarm from another node due to an alarm propagation;

(v) Underrequest Monitoring Mode (UMM): a central node can send a request to a sensor node to collect environment data immediately;

(vi) Data Dump Mode (DDM): this mode is devoted to collect a set of data previously stored at the node memory.

2.3. Sensor Network. Although all SNs have the same hardware, they can have different functionalities and roles in the field network. The field network has a tree structure, with a variable number of tiers. There are three different types of nodes according to their role in the network and their functionality. Type A nodes are the ones at the top of the tree, directly connected to a $\mathrm{CN}$ through a serial cable. They also provide a radio interface to communicate with the rest of the field network. Type B nodes only have a radio interface to communicate. Apart from taking their own measurements, they are able to reroute information packets in both directions of the network, from bottom to top and from top to bottom. Lastly, type $C$ nodes are plain sensor nodes without routing capabilities that send their measures to the nearest router node. This SN network structure is shown in Figure 6.

The process of setting up the field network is automatic. End nodes can be deployed and turned on without having to follow any specific sequence. All the nodes carry out an autodiscovery protocol when they are first started. When completed, this discovery protocol results in the formation of the tree structure, in which every node has a "routing parent" and a link to its corresponding $\mathrm{CN}$.

All the nodes in the network take part in a keep-alive scheme, in order to detect broken links or dead neighbours. If the keep-alive constraint is not fulfilled for a particular node, it restarts its autodiscovery functionality in order to find a new "routing parent." This way, the network topology is dynamically updated. There has to be some network redundancy in terms of $\mathrm{CN}$ to guarantee that the system keeps delivering data despite an assigned $\mathrm{CN}$ failed, by rerouting information to another neighbouring $\mathrm{CN}$. In the unlikely event that all the CNs fail in a network branch, it would be detected by a no-reply scenario within a specified period of time (host unreachable), probably requiring direct maintenance tasks if it is not due to a fire itself.

All the SNs employ a bidirectional communication scheme. They can receive commands to change alarm thresholds or node working mode. The communications protocol includes the following communication message types:

(i) search messages: search for an initial node link;

(ii) automatic data messages: periodic messages containing sensor data;

(iii) specific data messages: messages containing sensor data in response to a higher level node request; 


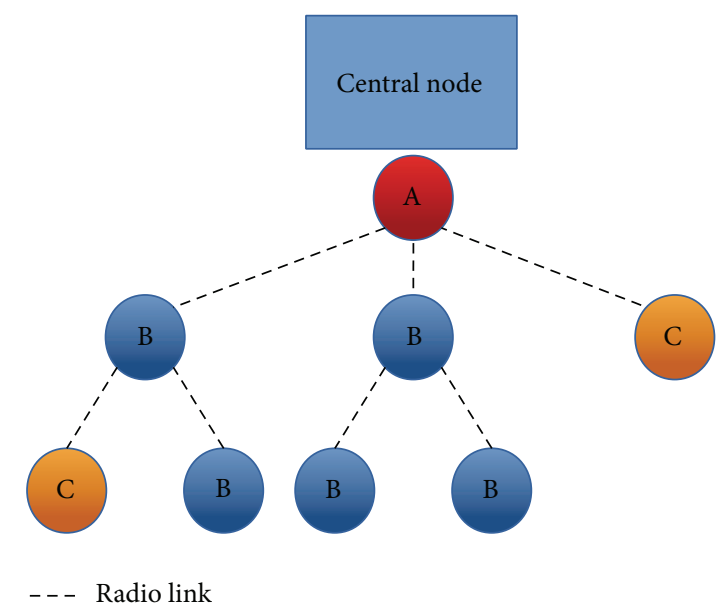

FIGURE 6: Field network tree structure. A: nodes are directly connected to central nodes. B: nodes have sensing and routing functionalities. C: nodes only have sensing capabilities.

(iv) node status messages: messages containing data regarding sensor status, power level, memory availability, additional status, and so forth;

(v) alarm messages: not only the alarm messages themselves, but also the bidirectional confirmation messages (error checking messages) to ensure that the alarm message has been properly transmitted and received;

(vi) command messages: messages from higher level nodes with commands;

(vii) rerouting messages: message hopping corresponding to other messages from other nodes.

Each SN has two types of links: a physical link, to a nearby node for rerouting, and a logical link, to a $\mathrm{CN}$ from which it depends. There will be an internal record to store the identification of the SNs whose data have to be echoed in an ascending or descending way. When new nodes are added to the network, id of each available node (in range) will also be stored at each node. At the end of each data path, there will be a $\mathrm{CN}$ with long range communication capabilities.

Finally, time synchronization is essential in WSN applications to keep communications ordered in the shared channel and to correlate the measurements taken with a time-stamp. This feature also leads to save power in most devices because if one node is aware of the fact that it is not going to transmit or receive any frame for some time, it may switch its radio module off. All SNs have a very low power real time clock which manages this time base. CNs, which have GPS capability, provide time information and also have the capability to detect time drifts from other nodes. When this problem is detected, a clock correction frame is sent to the drifted node.

\section{Experiments and Results}

Different experiments were aimed at ensuring that the network functioning and integration with the rest of the systems were in accordance with the expected performance. The two CNs linked together correctly at a distance of $1.6 \mathrm{~km}$. The SNs were located at $25 \mathrm{~m}$ of distance among them approximately. Their data was transferred to the middleware successfully by means of the $\mathrm{CN}$ connected to the computer at the Control Centre. In particular, consider the following:

(i) ISM range performance: as it is necessary to provide network communications to remote wildland locations far from urban environments, with no GSM coverage, the ISM long range interface must be able to cover distances of several kilometers. The main central nodes were recommended to be fixed at a previously studied location and then they dynamically place the secondary nodes at different line of sight locations as shown in Figures $8(\mathrm{a})$ and $8(\mathrm{~b})$. The aim of this procedure was to assess the possible link degradation with range distance and how the nodes automatically choose the most efficient power configuration. The link quality indicator (LQI) was the metric employed to measure the percentage of successful received packets. In our experiments, the best quality levels were obtained with links P1 and $\mathrm{P} 4$, with LQI $=100 \%$ and $97 \%$, respectively. $\mathrm{P} 2$ and P3 showed a similar LQI value, around $80 \%$. Missing packets were just transmitted again until an acknowledge message was received;

(ii) fire detection performance: we computed the fire indexes according to the Fire Weather Index (FWI) System. The nodes take into account this index and the evolution of the raw measurements like abnormal temperature increments, humidity decrements, or gas detection, to generate an alarm message. Different fire 


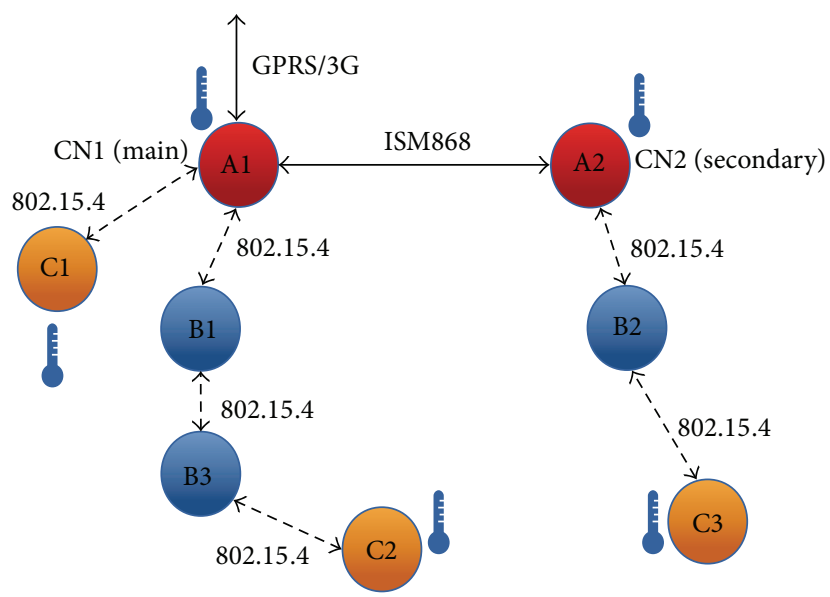

Figure 7: Network structure used in the pilot study.

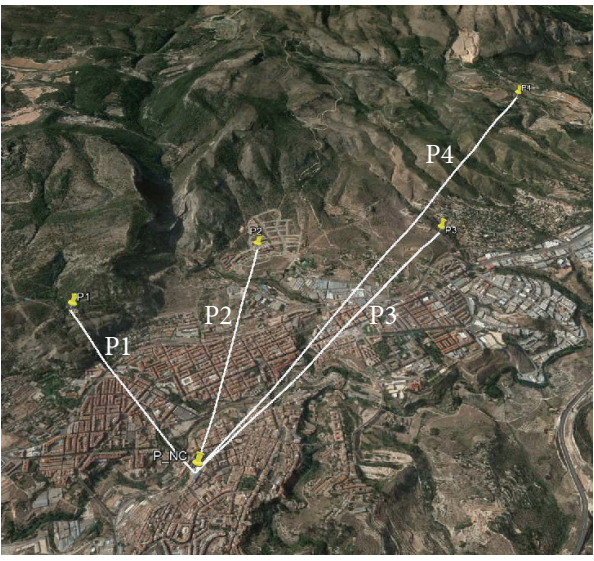

(a)

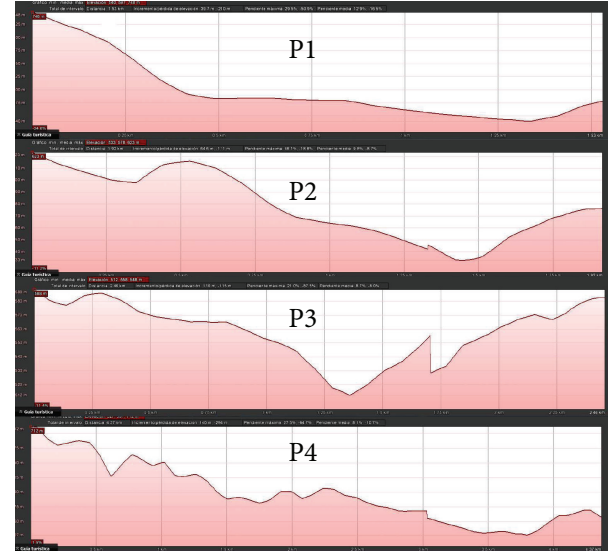

(b)

FIGURE 8: Tests carried out to check ISM communication performance between central nodes. In (a), the line routes between both nodes are shown, where distances are P1: $1.4 \mathrm{~km}, \mathrm{P} 2: 2.2 \mathrm{~km}, \mathrm{P} 3: 3.3 \mathrm{~km}$, and P4: $4.8 \mathrm{~km}$. In (b), the elevation map of these routes is shown. As it can be seen, all routes have line of sight.

scenarios were generated in a laboratory environment to detect real fires and check if false alarms were also raised. For that purpose, e-match pyrotechnic electric igniters were used under controlled conditions. Results exhibited a 100\% fire detection accuracy based on sensed temperature increasing more than 5 in less than 10 minutes and relative humidity decreasing more than $10 \%$ in the same period. The gas sensors detected in all cases significant smoke changes during the tests (concentration changes greater than 3\%);

(iii) final network deployment: the network proposed was tested under realistic conditions in the natural park of Sierra Mariola, Alcoy (Spain), in the context of a pilot study including all the key actors involved in wildland fire fighting operations. The network was composed of the following items:

(a) one main $\mathrm{CN}$ with a GPRS/3G interface to connect to the computer at the Control Centre, (b) one secondary $\mathrm{CN}$ linked to the main one by an ISM868 interface,

(c) six SNs, classified in accordance with their data routing capabilities: 3 SNs "B" type, with routing capabilities, and 3 SNs "C" type without such capabilities,

(d) a computer for network control, equipped with a $3 \mathrm{G}$ stick modem,

(e) a computer to collect data from the middleware by a subscription process,

(f) three wind sensors for the CNs and one of the SNs,

(g) all the nodes having temperature and humidity sensors.

This structure is depicted in Figure 7. Additional deployed items were a satellite communications network and a Wi-Max link between the Control Centre and the coordination aircraft. The network was used to collect humidity, temperature, 
and wind data in real time and send them to the computer at the command centre by means of the middleware. Results show that more than $95 \%$ of the taken measurements arrived to the Control Centre. Sensors and communication links were functioning perfectly and fire conditions were simulated in several nodes and alarms arrived immediately to all the actors in forest fire fighting operations.

\section{Discussion and Conclusions}

Forest fires are a very serious problem in many countries, and global warming may contribute to make this problem worse. Experts agree that, in order to prevent these tragedies from happening, it is necessary to invest in new technologies and equipment that enable a multifaceted approach [7].

This paper describes a WSN for early detection of forest fires. This network can be easily deployed at areas of special interest or risk. There are two types of nodes from the physical structure point of view: SNs, to collect data from the environment, and CNs, to gather data from the SNs and transmit the information to a Control Centre.

The nodes also can be in different functioning modes. This enables a proper and seamless configuration of the network, provides redundancy, and ensures there will be full temporal and geographical coverage in the deployment zone. The information gathered is related not only to early detection purposes but also to environment monitoring to maximize the WSN usage. This environmental data can also be employed to fire fighting preventive tasks such as vegetation modeling, microclimate studies, and propagation model parametrization.

In addition to the baseline functionality of a complete WSN, some experiments were developed to demonstrate that

(i) fire detection is very accurate and false alarms are rare, if any;

(ii) long range communications, which are critical, perform as expected. Radio links, regardless of the distance, are better if there is a direct line of sight. This effect is explained by the Fresnel zone;

(iii) realistic and successful field tests during two complete forest fire fighting operations involve all the key players (aerial and land firefighting, command centre, coordination, and satellite imagery) in the Mariola Natural Park in Alcoy (Spain) during February and October 2013;

(iv) customised communications protocol employs short messages, maximizes sleeping stage duration, and minimizes power consumption;

(v) WSN is integrated with a middleware layer to transfer data to a Control Centre or command centre.

\section{Conflict of Interests}

The authors declare that there is no conflict of interests.

\section{Authors' Contribution}

Antonio Molina-Pico developed the central nodes and David Cuesta-Frau devised the PROMETEO project, led the task of developing the central nodes, and wrote the paper. Alvaro Araujo and Alba Rozas developed the sensor nodes and wrote the corresponding part of the paper. Javier Alejandre coordinated the development of the network and wrote the reporting deliverables for the PROMETEO project.

\section{Acknowledgment}

This work has been developed under the framework of the research project PROMETEO, CEN-20101010, funded by the Centre for the Technological Industrial Development (CDTI), Spanish Ministerio de Economia y Competitividad.

\section{References}

[1] M. Hefeeda and M. Bagheri, "Forest fire modeling and early detection using wireless sensor networks," Ad-Hoc and Sensor Wireless Networks, vol. 7, no. 3-4, pp. 169-224, 2009.

[2] Z. Li, S. Nadon, and J. Cihlar, "Satellite-based detection of Canadian Boreal forest fires: development and application of the algorithm," International Journal of Remote Sensing, vol. 21, no. 16, pp. 3057-3069, 2000.

[3] J. Zhang, W. Li, N. Han, and J. Kan, "Forest fire detection system based on a ZigBee wireless sensor network," Frontiers of Forestry in China, vol. 3, no. 3, pp. 369-374, 2008.

[4] Y. G. Sahin and T. Ince, "Early forest fire detection using radioacoustic sounding system," Sensors, vol. 9, no. 3, pp. 1485-1498, 2009.

[5] J. Lloret, M. Garcia, D. Bri, and S. Sendra, "A wireless sensor network deployment for rural and forest fire detection and verification," Sensors, vol. 9, no. 11, pp. 8722-8747, 2009.

[6] U. Ganesh, M. Anand, S. Arun, M. Dinesh, P. Gunaseelan, and R. Karthik, "Forest fire detection using optimized solarpowered Zigbee wireless sensor networks," International Journal of Scientific and Engineering Research, vol. 4, pp. 586-596, 2013.

[7] Government response to the Bushfires Royal Commission implementation monitor, Annual Report, 2014, https://www .emv.vic.gov.au/. 


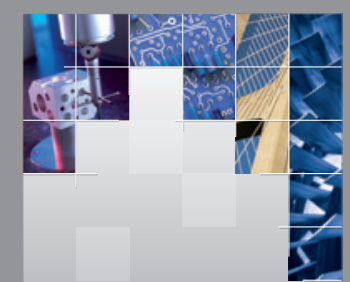

\section{Enfincering}
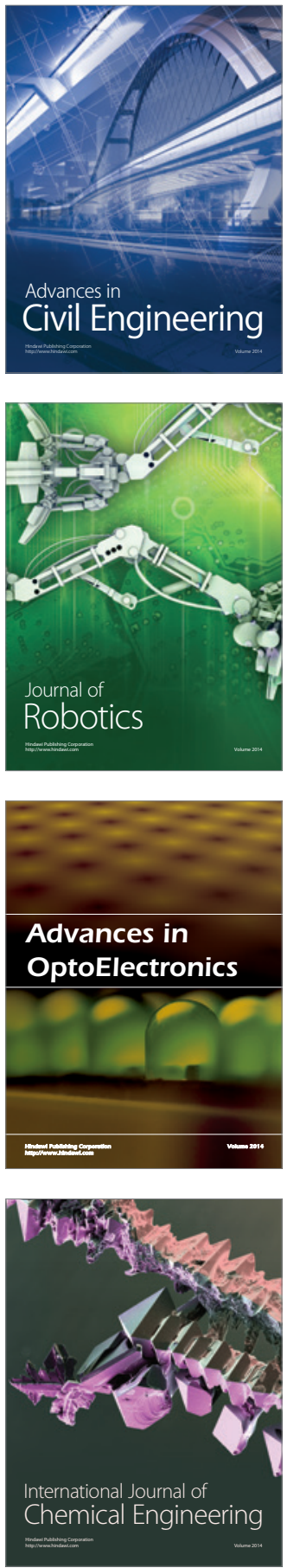

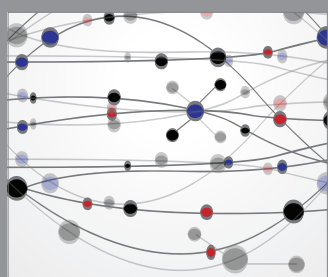

The Scientific World Journal

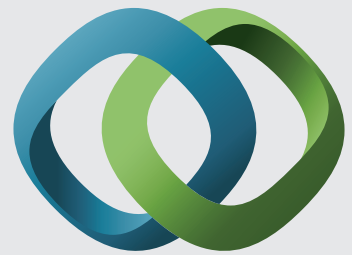

\section{Hindawi}

Submit your manuscripts at

http://www.hindawi.com
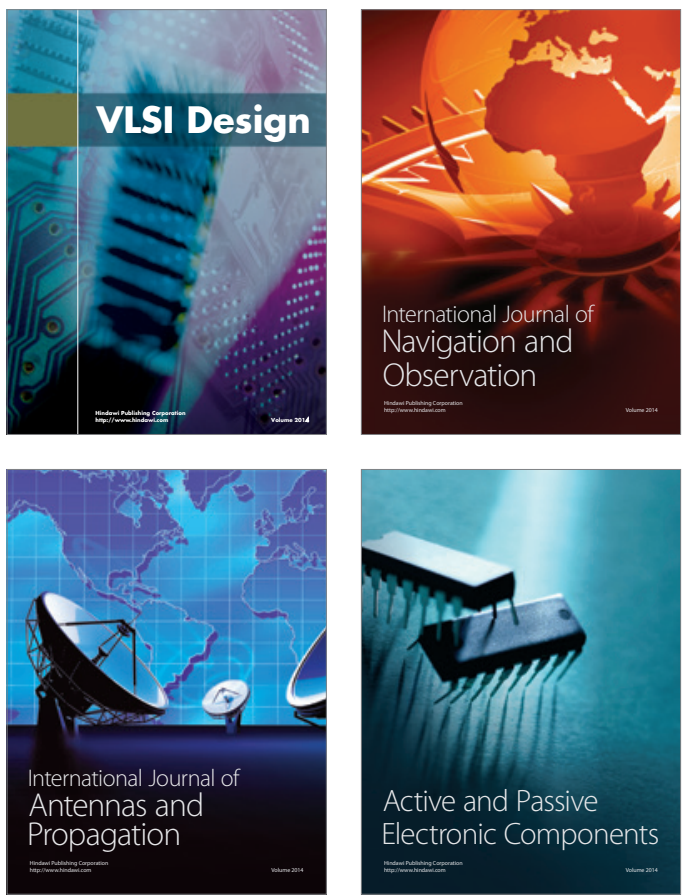
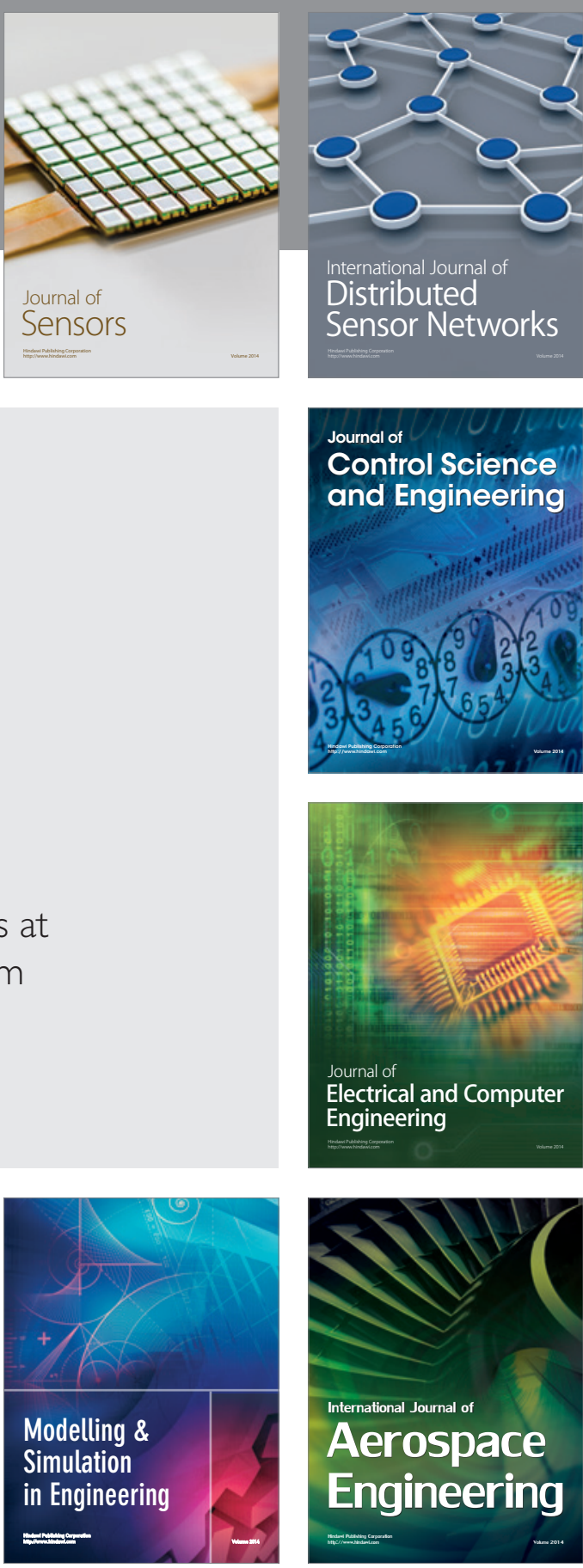

International Journal of

Distributed

Sensor Networks

Journal of

Control Science

and Engineering
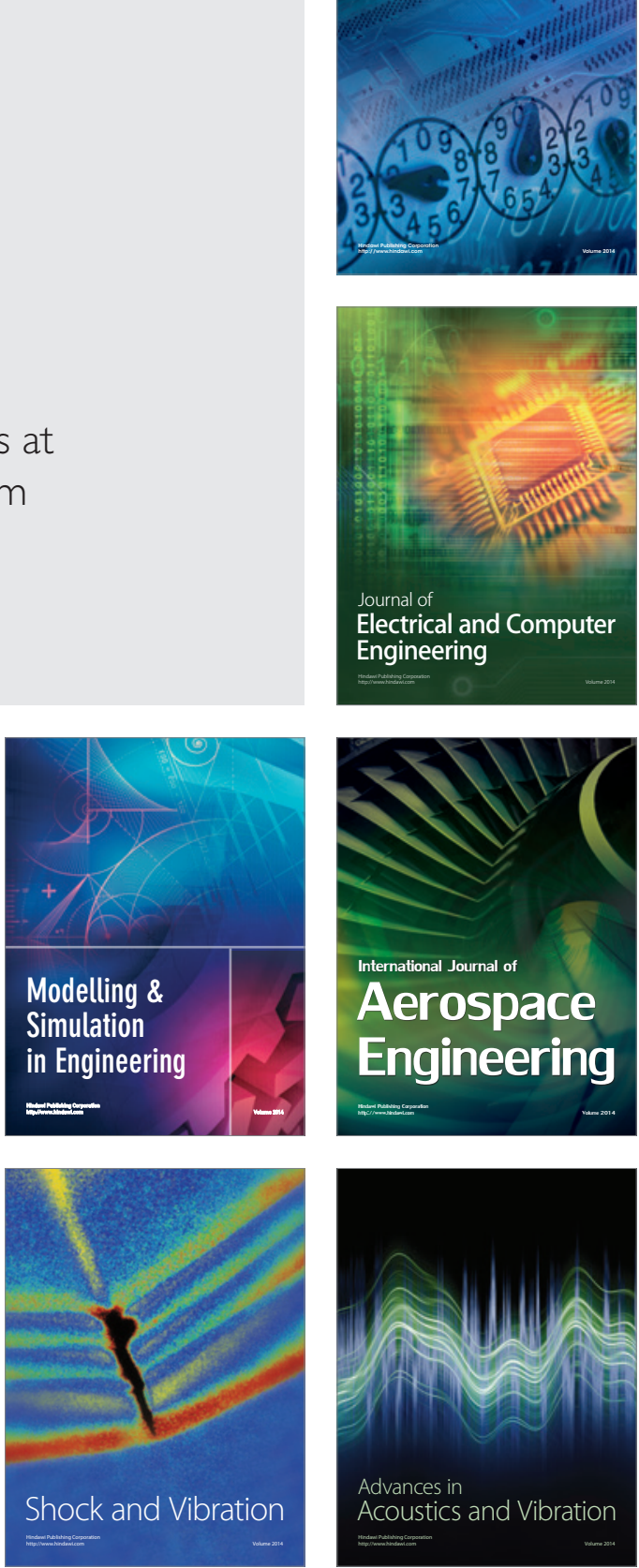